\title{
Kidney manifestation of melioidosis: a study from a tertiary care hospital in
} South India

\author{
ARPrabhu $^{1}$, TShaw $^{2}$, NSPrasad $^{1}$, RDarshan $^{1}$, CTellapragada $^{2}$, KEVandana $^{2}$, CMukhopadhyay $^{2}$ \\ Introduction \\ Melioidosis is caused by the facultative intracellular Gram-negative bacterium, \\ Burkholderiapseudomallei clinically ranging from subclinical infection to pneumonia or skin \\ involvement. The disease can involve any organ. Diabetes mellitus and chronic kidney disease \\ (CKD) are risk factors. We describe the renal manifestations of melioidosis in our population \\ and analyze their impact on disease outcome.
}

\section{Methods}

Consecutive culture confirmed melioidosis patients were recruited from January 2006 to December 2016 and analyzed. Chronic kidney disease was defined as evidence of kidney damage or estimated glomerular filtration rate (GFR) less than $60 \mathrm{~mL} / \mathrm{min} / 1.73 \mathrm{~m} 2$ for three months or more. Acute kidney injury (AKI) was defined as an increase in serum creatinine by $0.3 \mathrm{mg} / \mathrm{dL}$ or more within 48 hours or increase in serum creatinine to 1.5 times baseline or more within the last 7 days or urine output less than $0.5 \mathrm{~mL} / \mathrm{kg} / \mathrm{h}$ for 6 hours. An estimated GFR $75 \mathrm{~mL} / \mathrm{min} / 1.73 \mathrm{~m} 2$ was considered as normal. Basic demographics of the study population were studied and the associations of the renal dysfunction were analyzed for disease outcome and presentation.

\section{Results}

Of 200 individuals with melioidosis 34 (17\%) had renal impairment including 21(10.5\%) with CKD and AKI in 16 (8\%). Mean age was $46.6 \pm 15.8$ years and 160 (80\%) were male. Both CKD and AKI were associated with an increased chance of developing bacteremia $(\mathrm{p}=0.001)$.Mortality was seen in seven $(33.3 \%)$ with CKD $(\mathrm{p}<0.001)$ and in seven $(43.7 \%)$ with AKI (p<0.001). Sepsis with AKI was seen in $(28.5 \%)(\mathrm{p}<0.001)$.

\section{Conclusion}

Melioidosis causes AKI in $8 \%$ and kidney dysfunction predisposes to bacteremia and mortality.

\footnotetext{
${ }^{1}$ Department of Nephrology, Kasturba Medical College, Manipal University, Manipal, India ${ }^{2}$ Department of Microbiology, Kasturba Medical College, Manipal University, Manipal, India Address for correspondence: Prof. RavindraPrabhu, Department of Nephrology, Kasturba Medical, College, Manipal University, Manipal, India +919448107771Email: ravindra.prabhu@manipal.edu (D) https://orcid.org/0000-0002-5980-7197
} 